\title{
Attaques des arbres par les termites sur le campus de l'Université de Lomé (Togo)
}

\author{
Essè ANANI KOTOKLO*, Boris Dodji KASSENEY, Wolali NYAMADOR, Guillaume \\ Koffivi KETOH et Adolé Isabelle GLITHO
}

Laboratoire d'Entomologie Appliqué, Faculté des Sciences, Université de Lomé, BP : 1515 Lomé (Togo).

"Corresponding author,E-mail: akejacques07@yahoo.fr, Tél : (00228)9189929 /(00228)2347642 /

$$
\text { (00228)2255094 Poste } 1321
$$

\section{RESUME}

L'étude menée sur les attaques des arbres par les termites sur le campus de l'Université de Lomé (Togo) a montré que 304 plants (92,40\% de peuplement d'arbres répartis en 20 espèces) ont été attaqués par les termites. Les attaques des termites sur ces arbres se manifestent par la couverture des troncs et des branches par des galeries-tunnels et des placages de récolte des termites, par des racines, des écorces, du bois consommé entraînant la chute des feuilles, le dépérissement et/ou la mort de la plante. Les espèces de termites responsables des dommages causés aux arbres appartiennent au groupe trophique des xylophages. Il s'agit principalement de 5 espèces appartenant à 3 genres : Amitermes evuncifer Silvestri, Ancistrotermes cavithorax Sjöstedt, Ancistrotermes guineensis Silvestri, Macrotermes bellicosus Smeathman et Macrotermes subhyalinus Rambur. Les résultats de notre étude constituent un préliminaire indispensable pour la protection des arbres sur le campus contre les attaques des termites.

() 2010 International Formulae Group. All rights reserved.

Mots clés : Campus, université, Lomé, Togo, termites, dommages.

\section{INTRODUCTION}

L'ordre des Isoptères ou les termites a été pendant longtemps considéré comme consommateurs de bois morts et leurs principaux dégâts se situent au niveau des bois ouvrés. Depuis quelques décennies, une attention particulière est portée sur l'étude des termites, compte tenu de leur intérêt économique. En effet, bien que ces insectes représentent une nuisance majeure pour les agriculteurs des régions intertropicales, notamment l'Afrique qui compte plus du tiers des espèces existant sur le globe, leur impact étant néanmoins bénéfique sur les propriétés physiques et chimiques des sols. De nombreuses espèces de termites ont été signalées en Afrique comme ravageurs d'essences forestières (Agbogba et Roy-Noël, 1982), fruitières (Ndiaye et Han, 2002) et domestiquées (Renoux, 1997).

Les dégâts causés par les termites aux cultures et aux plantations sont souvent supérieurs à $15 \%$ et peuvent atteindre parfois 90\% (Wood et Pearce, 1991). Leurs dommages et les coûts des réparations ont été évalués à plus de 22 milliards d'euros chaque année (Fuchs et al., 2004). 
$\mathrm{Au}$ Togo, il existe quelques travaux sur l'inventaire des termites, leurs habitats et leurs dégâts (Anani Kotoklo, 2000, 2007); et sur le contrôle de leurs populations (Kétoh, 2004). Mais nous n'avons recensé aucune étude sur les dommages causés aux essences végétales par les termites. Sur le domaine de l'Université de Lomé, des colonies de termites prolifèrent à un rythme rapide et le nombre des termitières est en croissance presque exponentielle. Cette pullulation de termites et de leurs nids n'est pas sans conséquence néfaste sur la production et le développement des arbres. En général, les termitières constituent la source des infestations (Aisagbonhi, 1989). Il est donc urgent pour un bon développement et une protection des essences ligneuses de répertorier les espèces ravageuses et de quantifier sur le domaine universitaire leurs impacts sur les arbres en vue d'une lutte programmée.

Le présent travail vise à faire l'inventaire des termites ravageurs des arbres sur le campus de Lomé et à évaluer leur impact sur le développement de ces arbres.

\section{MATERIEL ET METHODES}

\section{Site d'étude}

Les études ont été réalisées sur le domaine universitaire de Lomé qui couvre une superficie totale de 248 ha. Le campus jouit d'un climat subéquatorial de type guinéen caractérisé par des températures variant très peu au cours de l'année (moyennes annuelles $28 \pm 2{ }^{\circ} \mathrm{C}$ ) et par un taux d'humidité relative moyen élevé $(80 \pm 10 \%$ par an). La pluviométrie annuelle moyenne varie entre 700 et $800 \mathrm{~mm}$. Le couvert végétal sur le campus est constitué en grande partie de Heteropogon contortus L. (Poacée) et de nombreuses plantes ligneuses implantées pour l'ombrage.

Le campus est bâti sur un plateau de sols rouges qui repose sur deux séries séparées par une discordance: la série marine sur laquelle se trouve la série continentale appelée «Continental terminal». Le campus est donc construit sur un sol ferrallitique caractérisé par une granulométrie sablo-argileuse (Duchaufour, 1995).

\section{Méthode d'évaluation des attaques de termites sur les arbres}

Des relevés ont été effectués dans quatre placeaux (100 m x $100 \mathrm{~m})$, en liaison avec l'abondance des espèces végétales sur le campus. L'observation a porté sur la présence des signes d'attaques des termites et l'état des arbres sur une hauteur de 2,50 m du sol. Les termites ont été recherchés dans les galeries et placages, sur le tronc et les branches, sur les racines apparentes. A la fin des observations, une échelle de cotation des attaques des termites sur les arbres a été établie (Tableau 1).

Des clés de détermination et divers ouvrages ont été utilisés pour l'identification des termites échantillonnés (Grassé, 1986; Bouillon et Mathot, 1965; Sands, 1992 ; Ruelle, 1970 ; Noirot, 2001). Les échantillons identifiés ont été confirmés par le laboratoire de l'UMR/CNRS 5548 de l'Université de Bourgogne, Dijon (France).

\section{RESULTATS \\ Inventaire systématique}

Dans les placeaux, nous avons recensé 329 arbres se répartissant en 20 espèces (Tableau 2). Parmi ces arbres, Azadirachta indica (A.) Juss. a le nombre de pieds le plus important (78), suivi de Delonix regia Raf., Eucalyptus tereticornis Sm. et de Cassia siamea Lam. avec respectivement 36, 33 et 30 pieds. Ces derniers constituent les plantes d'ombrage les plus plantées sur le campus

Sept espèces et 2 genres de termites ont été recensés sur ces arbres et se répartissent entre deux familles (Tableau 3): les Rhinotermitidae avec la sous-famille des Coptotermitinae et les Termitidae avec les sous-familles des Termitinae et Macrotermitinae. 
Tableau 1 : Echelle de cotation des attaques de termites sur les arbres.

\begin{tabular}{cl}
\hline Cotation & \multicolumn{1}{c}{ Définition des cotations } \\
\hline $\mathbf{E}_{\mathbf{0}}$ & Pas de galerie, ni de placage terreux, ni de présence de termite sur l'arbre : arbre sain \\
\hline $\mathbf{E}_{\mathbf{1}}$ & Présence de galeries et de placage terreux de termite sur l'écorce de l'arbre \\
\hline $\mathbf{E}_{\mathbf{2}}$ & Présence de termites sur et dans l'écorce de l'arbre \\
\hline $\mathbf{E}_{\mathbf{3}}$ & Présence de termites dans le bois de l'arbre \\
\hline $\mathbf{E}_{\mathbf{4}}$ & $\begin{array}{l}\text { Bois rongé par les termites et remplacé ou non par de la terre d'une longueur inférieure } \\
\text { à } 50 \mathrm{~cm}\end{array}$ \\
\hline $\mathbf{E}_{\mathbf{5}}$ & $\begin{array}{l}\text { Bois rongé par les termites et remplacé ou non par de la terre d'une longueur } \\
\text { supérieure à } 50 \mathrm{~cm} \text { avec mort de l'arbre }\end{array}$ \\
\hline
\end{tabular}

Tableau 2 : Répartition des espèces végétales dans les placeaux sur le campus.

\begin{tabular}{lc}
\hline Espèces végétales & Nombre de pieds \\
\hline Casuarina equistifolia L. & 5 \\
Acacia auriculiformis A. & 20 \\
Adansonia digitata L. & 2 \\
Azadirachta indica (A) Juss. & 78 \\
Blighia sapida C. & 6 \\
Cassia siamea Lam. & 30 \\
Callitris intratropica Barker (Sm.) & 5 \\
Cycas circinalis Roxb. & 3 \\
Citrus sinensis (L.) Osbeck. & 7 \\
Cocos nucifera L. & 5 \\
Delonix regia Raf. & 36 \\
Elaeis guineensis Jacq. & 26 \\
Eucalyptus tereticornis Sm. & 33 \\
Eucalyptus torelliana Muell. & 10 \\
Gmelina arborea Roxb. & 15 \\
Khaya senegalensis (A.) Juss. & 3 \\
Mangifera indica L. & 18 \\
Sterculia foetida L. & 12 \\
Terminalia catappa L. & 13 \\
Terminalia mantaly L. & 2 \\
\hline
\end{tabular}

Sur le plan trophique, les espèces de termites récoltées sur des arbres au campus appartiennent au seul groupe des xylophages. Ils se subdivisent en deux types: les xylophages stricts, appelés également lignivores et les xylophages champignonnistes ; les premiers se nourrissent de bois sous toutes ses formes, y compris les résidus en décomposition dans la litière. Ils appartiennent aux sous-familles des Coptotermitinae et Termitinae. Le second type est celui des xylophages champignonnistes. Ce groupe comprend les consommateurs de bois et de la litière. Ils regroupent les espèces appartenant à la sous-famille des Macrotermitinae. 


\section{Etude quantitative}

Un peuplement de 329 arbres a été examiné (Tableau 4). Sur cet ensemble, $92,40 \%$ des arbres ont présenté des attaques de termites, avec 3,8\% (13 arbres) de mortalité

\section{Etude qualitative}

Les espèces de termites ravageurs récoltés sur le campus sont inféodées chacune à plusieurs espèces ligneuses (Tableau 5) :

\section{Coptotermes intermedius Silvestri}

C. intermedius attaque 5 espèces végétales : Delonix regia Raf., Cassia siamea Lam., Callitris intratropica Barker (Sm), Eucalyptus torelliana Muell. et Gmelina arborea Roxb. Des ouvriers de cette espèce ont creusé plusieurs galeries-tunnels et des placages sur le tronc et les branches de ces arbres. Les attaques ne se sont pas seulement limitées à l'écorce mais se sont étendues également à l'intérieur de l'arbre sur le périderme et le bois. Certains de ces arbres (D. regia et $C$. siamea) sont fortement attaqués et leurs troncs sont totalement couverts de galeries-tunnels et de galeries de récolte. Souvent, ces galeries partent des racines apparentes et remontent vers les branches. Des ouvriers, des soldats et des larves de $C$. intermedius ont été trouvés dans certaines galeries de récolte mises à nu.

Amitermes evuncifer Silvestri
Les attaques de cette espèce se font sur l'écorce ou dans le bois de Delonix regia Raf., Azadirachta indica (A) Juss. Mangifera indica L. Casuarina equisetifolia L., Eucalyptus tereticornis Sm. à l'abri de galeries ou de placages. Les galeries partent en grande partie $\mathrm{du}$ sol et remontent vers les branches en passant par le tronc de l'arbre. Les attaques les plus fréquentes qui atteignent rapidement le bois et cambium de l'arbre se font à la faveur de fissures ou de petits trous sur le tronc. Des nids de A. evuncifer se localisent à la base de certains arbres sur le sol près des racines. Les attaques de cette espèce sur $M$. indica provoquent un écoulement de sève. C'est l'une des espèces la plus dévastatrice de $M$. indica sur le campus.

\section{Microcerotermes fuscotibialis Sjöstedt}

M. fuscotibialis a été rencontré sur Citrus sinensis (L.) Osbeck. avec la présence d'un nid arboricole. Plusieurs galeries-tunnels en relation avec le sol longent le tronc et les branches des arbres attaqués. Dans ces galeries circulent les termites. Les attaques observées se limitent à l'écorce des arbres.

\section{Macrotermes spp.}

M. bellicosus et M. subhyalinus emprisonnent certains arbres attaqués par la construction de nids en forme de manchon autour des racines principales et des troncs à quelques centimètres au dessus du sol. De l'intérieur des termitières, ces termites

Tableau 3 : Liste des espèces de termites rencontrées sur les arbres.

\begin{tabular}{|c|c|c|}
\hline Famille & Sous-famille & Espèce \\
\hline Rhinotermitidae & Coptotermitinae & Coptotermes intermedius Silvestri 1912 \\
\hline \multirow{9}{*}{ Termitidae } & Termitinae & Amitermes evuncifer Silvestri 1912 \\
\hline & & Microcerotermes fuscotibialis Sjöstedt 1896 \\
\hline & & \\
\hline & & Macrotermes bellicosus Smeathman 1781 \\
\hline & & Macrotermes subhyalinus Rambur 1842 \\
\hline & Macrotermitinae & Microtermes sp. \\
\hline & & Ancistrotermes cavithorax Sjöstedt 1899 \\
\hline & & Ancistrotermes guineensis Silvestri 1912 \\
\hline & & Allodontermes sp. \\
\hline
\end{tabular}


Tableau 4: Répartition du nombre d'arbres attaqués et du taux d'attaque dans chaque échelle de cotation.

\begin{tabular}{|c|c|c|}
\hline Echelle de cotation & Nombre d'arbres & Taux d'attaque (\%) \\
\hline $\mathrm{E}_{0}$ & 25 & $7,60^{*}$ \\
\hline $\mathrm{E}_{1}$ & 194 & 58,97 \\
\hline $\mathrm{E}_{2}$ & 81 & 24,62 \\
\hline $\mathrm{E}_{3}$ & 10 & 2,98 \\
\hline $\mathrm{E}_{4}$ & 6 & 1,79 \\
\hline $\mathrm{E}_{5}$ & 13 & 3,87 \\
\hline Total & 329 & 100 \\
\hline $\begin{array}{l}\text { Nombre d'arbres infestés par les } \\
\text { attaques de termite }\end{array}$ & 304 & 92,40 \\
\hline
\end{tabular}

Tableau 5 : Espèces ligneuses attaquées par les termites.

\begin{tabular}{ll}
\hline Espèce de termites & Essences attaquées \\
\hline Coptotermes intermedius & $\begin{array}{l}\text { Delonix regia, Cassia siamea, Callitris intratropica, Eucalyptus } \\
\text { toreliana, Gmelina arborea }\end{array}$ \\
\hline Amitermes evuncifer & $\begin{array}{l}\text { Delonix regia, Azadirachta indica, Mangifera indica, Casuarina } \\
\text { equisetifolia, Eucalyptus tereticornis }\end{array}$ \\
\hline Microcerotermes fuscotibialis & Citrus sinensis \\
\hline Macrotermes bellicosus & $\begin{array}{l}\text { Delonix regia, Azadirachta indica, Mangifera indica, Acacia } \\
\text { auriculiformis, Gmelina arborea }\end{array}$ \\
\hline Macrotermes subhyalinus & $\begin{array}{l}\text { Delonix regia, Mangifera indica, Cassia siamea, Eucalyptus } \\
\text { tereticornis, E. torelliana }\end{array}$ \\
\hline Microtermes sp & $\begin{array}{l}\text { Cassia siamea, Elaeis guineensis, Cocos nucifera, Sterculia } \\
\text { foetida, }\end{array}$ \\
\hline Ancistrotermes cavithorax & $\begin{array}{l}\text { Azadirachta indica, Acacia auriculiformis, Blighia sapida, } \\
\text { Cycas circinalis, Gmelina arborea, Terminalia catappa, T. } \\
\text { mantaly Mangifera indica }\end{array}$ \\
\hline Ancistrotermes guineensis & $\begin{array}{l}\text { Delonix regia, Cassia siamea, Callitris intratropica, Gmelina } \\
\text { arborea, Casuarina equisetifolia, Eucalyptus tereticornis }\end{array}$ \\
\hline Allodontermes sp & Blighia sapida, Elaeis guineensis, Cycas circinalis \\
\hline
\end{tabular}

détruisent les grosses racines pour se nourrir. Ils rongent l'écorce et attaquent le bois de l'arbre entrainant la chute des feuilles et à terme, la mort de l'arbre. Des galeries ou des placages argileux mis à nu laissent voir des ouvriers de Macrotermes se déplacer activement sur les troncs des arbres attaqués.

\section{Ancistrotermes spp.}

A. cavithorax et A. guineensis attaquent plusieurs arbres sur le campus. Les attaques des deux espèces sont localisées au niveau de l'écorce des troncs et des branches des arbres, avec souvent de spectaculaires constructions de galeries et de placages argileux. Ils sont considérés comme responsables de la dégradation de l'écorce et de la consommation des parties mortes des arbres. Si ces attaques ne provoquent pas directement la mort de l'arbre, elles entravent considérablement la circulation de la sève. Sur certains arbres, l'attaque de ces termites peut provenir de l'intérieur. Dans ce cas les parties de l'arbre 
vidées sont comblées par de la terre. Leurs nids sont souterrains et non matérialisés à la surface du sol.

Microtermes sp. et Allodontermes sp.

Les attaques de ces deux genres ne se limitent qu'au niveau du système racinaire des arbres à la surface du sol. La consommation des racines laisse des traces de morsures avec des galeries de pénétration de petites tailles.

\section{DISCUSSION}

Dans cette étude, 7 espèces et 2 genres de termites responsables des attaques des arbres ont été recensées sur le campus de Lomé. Ces espèces et genres appartiennent aux sous-familles des Coptotermitinae, Termitinae et Macrotermitinae et sont tous des xylophages. Ils attaquent les arbres compte tenu de leur régime alimentaire principalement à base de cellulose et de leur besoin en eau. En Afrique tropicale, des travaux ont montré qu'en plus des Termitinae (Sands, 1973; Wood et al., 1980) et des Macrotermitinae (Mora et al., 1990 ; Wood et Pearce, 1991), des Nasutitermitinae (Pretorius et al., 1991 ; Mampouya, 1996) sont également responsables d'importants dommages causés aux cultures, aux plantations et aux essences forestières et fruitières ainsi qu'aux plantes horticoles. En outre, dans des travaux similaires, des auteurs ont signalé les attaques des termites sur plusieurs plantes. Selon Renoux (1997), la plupart des plantes africaines domestiquées subissent des attaques des termites: acacia, anacardier, cacaoyer, cassia, cocotier, citronnier, eucalyptus, goyavier, hévéa, mandarinier, manguier, oranger, palmier à huile, etc. Au Sénégal, dans la région de Casamance, vingt-trois espèces de termites ont été inventoriées sur des arbres fruitiers (Ndiaye et Han, 2002). Au Nigeria, sur le campus de Obafemi Awolowo, Université d'Ife, des termites ont causé d'importants dégâts sur des arbres ornementaux et d'ombrage (Malako, 1983). En Inde, plus de 40 espèces de plantes ornementales, d'arbres fruitiers et d'essences forestières sont attaquées par les termites (Parihar, 1981) et entre 1975 et 1977 sur le seul campus de Bangalore, 20 à 40\% des plants de manguiers sont attaqués par les termites (Veeresh et al., 1988).

Les dégâts de termites observés au cours de nos travaux vont du dépérissement à la mort des arbres, en passant par une simple couverture des galeries-tunnels et de placages de récolte sur la surface de l'écorce, la consommation des racines, des écorces, du bois et la chute des feuilles. Ainsi, l'action délétère des termites sur les arbres observés se traduit par des impacts d'attaques de termites laissés sur ces arbres. Cependant, cette action peut se traduire autrement chez l'arbre par la baisse de l'activité photosynthétique due à la couverture du tronc de l'arbre par des placages terreux. Logan et EL Bakri (1990) ont montré que la présence de galeries-tunnels et de placages de récolte sur l'arbre peut réduire sa capacité photosynthétique et lui causer du stress, source de futures attaques de termites.

Dans nos observations, le taux d'attaque dû aux termites est variable selon les arbres. Il en est de même pour le mode d'attaque qui diffère selon les espèces de termites. Cette variabilité pourrait être liée à la teneur en extraits (tanins, flavonoïdes, lignanes, stilbènes terpénoïdes, tropolones, etc.) et en lignine dont dépend la durabilité naturelle $\mathrm{du}$ bois face aux attaques des champignons et des insectes. Plusieurs espèces de termites sont présentes sur une même essence. Ceci semble indiquer que certaines essences seraient plus appétables que d'autres. Des observations similaires ont été faites par Roy-Noël (1974) ainsi que par Ndiaye et Han (2002) qui avaient déjà souligné que plusieurs espèces de termites se retrouvant sur les mêmes pieds d'arbres peuvent exploiter la même partie du végétal et entrer ainsi en compétition ou exploiter des régions anatomiques différentes du végétal. La proportion élevée d'arbres infestés $(92,40 \%)$ par les termites pourrait être liée à la nature $\mathrm{du}$ sol $\mathrm{du}$ campus (sol rouge 
ferrallitique) qui favoriserait l'installation des termitières, sources des infestations des plantes. Thakur et al. (1989) soulignent en Inde que l'incidence et l'intensité des attaques des termites varient suivant l'espèce, d'une localité à une autre, et suivant les différents climats et les conditions édaphiques.

\section{Conclusion}

Différentes espèces de termites s'attaquent aux arbres plantés sur le campus de Lomé. Ainsi ont été identifiés comme termites responsables des attaques de ces arbres: Coptotermes intermedius Silvestri, Amitermes evuncifer Silvestri, Microtermes fuscotibialis Sjöstedt, Macrotermes bellicosus Smeathman, M. subhyalinus Rambur, Microtermes sp., Ancistrotermes cavithorax Sjöstedt, A. guineensis Silvestri et Allodontermes sp. Les dommages causés aux arbres vont du dépérissement à la mort de certains de ces arbres.

Toutes les espèces répertoriées appartiennent à un seul groupe trophique, celui des xylophages. Parmi ceux-ci, nous avons les xylophages stricts (lignivores) et les xylophages champignonnistes. Des méthodes de lutte adéquates contre ces insectes déprédateurs doivent être entreprises pour un bon développement de ces arbres.

\section{REMERCIEMENTS}

Nous remercions sincèrement le Professeur Tano Yao du Département de Zoologie, UFR de Biosciences, Université de Cocody, Abidjan (Côte d'Ivoire) pour son appui scientifique et les Docteurs Guelly Kudzo Atsu, Woégan Yao Agbélésséssi ainsi que Monsieur Radji Raoufou Pierre du Laboratoire de Botanique et d'Ecologie Végétale, Université de Lomé (Togo) pour leur contribution dans l'identification des espèces végétales.

\section{REFERENCES BIBLIOGRAPHIQUES}

Agbogba C, Roy-Noël J. 1982. L'attaque des arbres par les termites dans la Presqu'île du Cap-vert (Sénégal). III. Cas du parc forestier de Dakar-Hann sur sable ogolien. Bull. l'IFAN, série. A, 44(3-4): 341-364.

Aisagbonhi CI. 1989. A survey of the destruction effect of Macrotermes bellicosus Smeathman (Isoptera: Termitidae-Macrotermitinae) on coconut seednuts at NIFOR, Benin, Nigeria. Trop. Pest. Manag., 35(4): 380-381.

Anani Kotoklo E. 2000. Note préliminaire sur les termitières du domaine universitaire de Lomé (Togo). Cah. Géol., 135: 18851889.

Anani Kotoklo E. 2007. Inventaire des termites et évaluation de leurs dégâts dans deux champs de cannes à sucre au sud du Togo. Thèse de Doctorat, Université de Lomé, p 231.

Bouillon A, Mathot G. 1965. Quel est ce termite africain ? Zooleo. $\mathrm{N}^{\circ}$ 1. (Ed). Université de Léopoldville, Léopoldville.

Duchaufour P. 1995. Pédologie: Sol, Végétation, Environnement (4 ${ }^{\mathrm{è}}$ édn). Paris.

Fuchs A, Schreyer A, Feuerbach S, Korb J. 2004. A new technique for termite monitoring using computer tomography and endoscopy. Intern. J. Pest Manag. 50: 63-66.

Grassé PP. 1986. Termitologia: Comportement, Socialité, Ecologie, Evolution, Systématique (Tome 3). Fondation Singer-Polignac, Masson : Paris.

Ketoh GK. 2004. Evaluation of three plants potentialities in field and house termite control in Togo. Rapport FIS.

Logan JWM, El Bakri A. 1990. Termite damage to date palms (Phoenix dactylifera L.) in northern Sudan with particular reference to the Dongola District. Trop. Sci., 30: 95-108.

Malako SLO. 1983. Economic importance of termites. Six cases studies in Nigeria and Ghana. The Nigeria Field, 47(4): 222230.

Mampouya D. 1996. Les populations de termites d'une culture de canne à sucre 
irriguée dans un écosystème sahélien; le fipronil dans une lutte ciblée contre les termites. Thèse d'Université, Université Paris XII Val de Marne (France).

Mora P, Rouland C, Dibangou V, Renoux J. 1990. Damage caused by the recent infestation of the sugar cane fields by the fungus-growing termites Pseudacanthotermes spiniger. In Social Insects and the Environment, Veeresh GK, Mallik B, Viraktamath CA (eds). Oxford I.B.H.: New-Delhi, India; 604-605.

Ndiaye AB, Han SH. 2002. Attaque des arbres fruitiers par les termites (Isoptera) en Casamance (Sénégal). Bull. Soc. Entomol. France, 107(2): 193-199.

Noirot CH. 2001. The gut of termites (Isoptera). Comparative anatomy, systematics, phylogeny. II Higher termites (Termitidae). Ann. Sc. Entomol., 37(4): 431-471.

Parihar DR. 1981. Some observations on distribution and pest status of termites attacking forestry plantations in the Rajasthan desert. Indian J. For. 4: 22-25.

Pretorius MW, Van Ark H, Mohr JD. 1991. Preliminary mound-fumigation trials for the control of Trinervitermes trinervoides colonies (Isoptera: Termitidae). Phytophylac., 23: 89-90.

Renoux J. 1997. Les termites et l'homme. Laboratoire EBENA, Université Paris XII Val de Marne. Paris ; 133-146.
Roy-Noël J. 1974. Recherches sur l'écologie des Isoptères de la Presqu'île du Capvert (Sénégal). II. Les espèces et leur écologie. Bull. l'IFAN, série A, 44(1-2): 115-146.

Ruelle J.E. 1970. A revision of the Termites of the genus Macrotermes from the Ethiopian Region (Isoptera: Termitidae). Bull. Bri. Mus. (Nat. Hist.) Entomol., 24(9): 365-444.

Sands WA. 1973. Termites as pests of tropical food crops. Pans., 19(2): 167-177.

Sands WA. 1992. The termite genus Amitermes in Africa and the Middle East. Natural Resources Institute (NRI) Bulletin, 51: 140.

Thakur ML, Kumar S, Negi A, Rawat DS. 1989. Chemical control of termites in Eucalyptus Hybrid. Indian Forester: 733-742.

Veeresh GK, Rajagopal D, Kumar NG. 1988. Management of termites in mango orchard. Act. Horticul., 231: 633-636.

Wood TG, Pearce MJ. 1991. Termites in Africa: The environmental impact of control measures and damage to crops, trees, rangeland and rural buildings. Sociobiol., 19(1): 221-234.

Wood TG, Smith RW, Johnson RA, Komolafe PO. 1980. Termite damage and crop loss studies in Nigeria. Pre-harvest loss to yams due to termites and other soil pests. Trop. Pest. Manag., 26: 255-378. 